\title{
Development of a new class of on-skin radio-sensors boosted by thin polymer-based batteries
}

\author{
M. C. Caccami and G. Marrocco \\ Department of Civil and Computer Science Engineering \\ University of Rome Tor Vergata \\ Rome, Italy \\ caccami@ing.uniroma2.it
}

\author{
M. P. Hogan ${ }^{1}$, M. Alfredsson ${ }^{1}$ and J. C. Batchelor ${ }^{2}$ \\ ${ }^{1}$ School of Physical Sciences, University of Kent \\ ${ }^{2}$ School of Engineering, University of Kent \\ Canterbury, Kent, UK
}

\begin{abstract}
Conductive polymers are currently collecting interest for the development of low-profile eco-friendly and biocompatible non-metallic batteries capable of providing a local power source for the next-generation flexible body-integrated electronics. In this contribution, we demonstrate the feasibility of an organic ultrathin and multilayered polymer-based battery integrating a radiofrequency identification (RFID) tag antenna by means of an electromagnetic characterization in UHF band of the polymeric films. The optimally modeling of the battery plus the antenna aimed to develop a new class of shape-conformable radiosensors suitable to adhere to the skin as a tattoo as well as a plaster. A prototype of the device was manufactured and its communication performances were characterized through the measurement of the realized gain of the tag attached directly onto a volunteer's skin.
\end{abstract}

Keywords-Conductive polymer, Organic battery, On-skin antenna, RFID technology.

\section{INTRODUCTION}

Recently, emerging Bio-integrated Electronic and Wearable Sensor Technologies have enabled the development of ultrathin, flexible and stretchable devices which are intimately mounted on the human body for various healthcare, entertainment and sports applications. Key challenges related to such a kind of devices still lie in the selection of biocompatible and soft materials that could mimic all the mechanical properties of the human body and in the choice of a power system to source the sensor activity. Batteries are one of the most widely explored compact forms of energy source for wearable applications. They have to meet the increasing demands of detecting multiple parameters simultaneously, while supporting data transmission as well as improving the communication performance of a tag antenna by increasing its read range [1]. The most currently common battery systems are based on $L i$-ion technology due to their high energy densities, long cycle life, good shelf-life, rapid charge and discharge capability, and high energy efficiency. A typical lithium ion battery consists of two metallic current collectors each placed on the anode/cathode. Such metallic current collectors allow for connection to an external circuit. Battery discharging/charging cycles involve the lithium ions migration between the two electrodes through an electrolyte solution. However, the battery and antenna are typically the bulkiest components in a transceiver system since they are manufactured using rigid and non-conformal materials. It is, therefore, of interest to combine them in order to create a low-profile integrated antenna/battery component. Moreover, it is well known that the antennas are subjected to mismatch and to efficiency reduction in the presence of proximal metal objects. Batteries thus should contain no metallic components, avoiding the standard use of a metallic hermetic enclosure. Therefore, the materials that replace the metallic elements should be sufficiently conductive to preserve the antenna performance [2].

Advances in material science and the novel digital fabrication technologies offer unprecedented options to realize low-profile batteries that are safer, eco-friendly, and can be lighter than a gram as well as thinner than a millimeter. In particular, conductive polymers are an important class of materials with outstanding properties such as reasonable electrical conductivity, good ionic conductivity, as well as easily manufacturing, making them a promising enabler of the development of green and biocompatible non-metallic thin film batteries [3] suitable to be integrated within an antenna.

This contribution addresses the development of a new class of Radiofrequency Identification (RFID) tag antennas mounted on skin and suitable to be integrated into the structure of a layered non-metallic polymer-based battery. In particular, a prototype has been designed and manufactured for the purpose of demonstrating the feasibility of an ultrathin battery integrating the antenna and using an organic conducting polymer as the anode material. Moreover, a preliminary electromagnetic characterization of the investigated conductive polymers was necessary to estimate in the UHF RFID band for the purpose of properly designing the on-skin antenna.

\section{CONDUCTIVE POLYMERS}

The most commonly used conducting polymers in the battery is the polyaniline (PANI), which was synthesized in laboratory, and (poly(3,4-ethylenedioxythiophene) polystyrene sulfonate (Pedot:PSS), which is a chemical interactive material (CIM), available commercially as a homogenized water based dispersion with a Pedot to PSS ratio of 1:2.5.

\section{A. Radiofrequency Characterization}

Very little knowledge is currently available about the radiofrequency features of conductive polymers. For the 
purpose of characterizing their unknown dielectric properties in the UHF band, the polymeric material films were deposited on two different substrates. Pedot:PSS was directly spread by spray coating over a $0.1 \mathrm{~mm}$-thin cellulose acetate sheet $\left(\varepsilon_{\mathrm{r}}=\right.$ 2.9 and $\sigma=0.059 \mathrm{~S} / \mathrm{m}$ ) whose rough side enabled the polymer to adhere, thus simplifying its deposition. N-methyl-2pyrrolidone (NMP) solvent was instead used to suspend the polyaniline's granules by ball milling. The PANI-NMP suspension was hence deposited over a $0.2 \mathrm{~mm}$-thin borosilicate glass layer $\left(\varepsilon_{\mathrm{r}}=4\right.$ and $\left.\sigma=2.9 \mathrm{e}^{-4} \mathrm{~S} / \mathrm{m}\right)$ by drop casting.

For the radiofrequency characterization of the dielectric properties of the conductive polymers in the UHF RFID band, the measurement set-up was based on a multilayer microstrip ring resonator, as in [4]. The samples were placed between the two layers of the ring resonator and measurements of the scattering parameter versus frequency were carried out by connecting the ports of the device to a Vector Network Analyzer. The dielectric properties of the polymeric films, permittivity and electrical conductivity, were then obtained in the UHF band through a combined experimental and numerical identification procedure involving the evaluation of the peak frequency $f_{R}$ of $S_{12}$ scattering parameter and accordingly the quality factor $\mathrm{Q}$ extracted from the measurements. The estimated values for the dielectric properties of the thin polymeric films highlight that in the UHF band Pedot:PSS exhibits a significant permittivity $\varepsilon_{\mathrm{r}}=82$ and a high electrical conductivity $\sigma=5.3 \mathrm{~S} / \mathrm{m}$ in ambient conditions and polyaniline has instead a value of permittivity $\varepsilon_{\mathrm{r}}>4$ in ambient conditions and is a lossy material with an electrical conductivity $\sigma$ close to $3 \mathrm{~S} / \mathrm{m}$.

Finally, a further electromagnetic characterization of the organic polymers was required under the hypothesis of the battery cycling process, which occurs when lithium ions $\mathrm{Li}^{+}$ migrate from the cathode to the anode material. The presence of $\mathrm{Li}^{+}$causes indeed an increase in both permittivity and electrical conductivity of the polymers. For instance, in the case of Pedot:PSS material, the permittivity increased by an order of approximately $10^{2}$ and the electrical conductivity approximately doubled (estimated values: $\varepsilon_{\mathrm{r}}=188.2$ and $\sigma=$ $10.4 \mathrm{~S} / \mathrm{m})$.

\section{ON-SKIN ANTENNA BOOSTED BY POLYMER-BASED BATTERY}

An antenna, suitable to be mounted on skin, was designed for integration into the structure of a layered polymer-based battery. The considered RFID tag is a nested slotline antenna derived from [5]. The geometry of the tag (Fig. 1) consists of a slot at the mid-length along the antenna, at a small distance $t$ from the upper edge. Two short coplanar lines separated by gap extend out of the slot center of the antenna to the RFID microchip (here EM4325 IC) which is connected across the two lines. A pad is

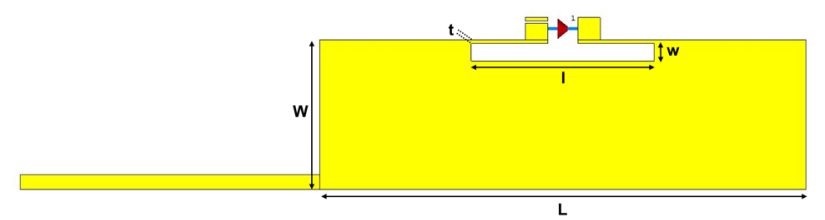

Fig. 1 Layout of the nested slotline RFID antenna.

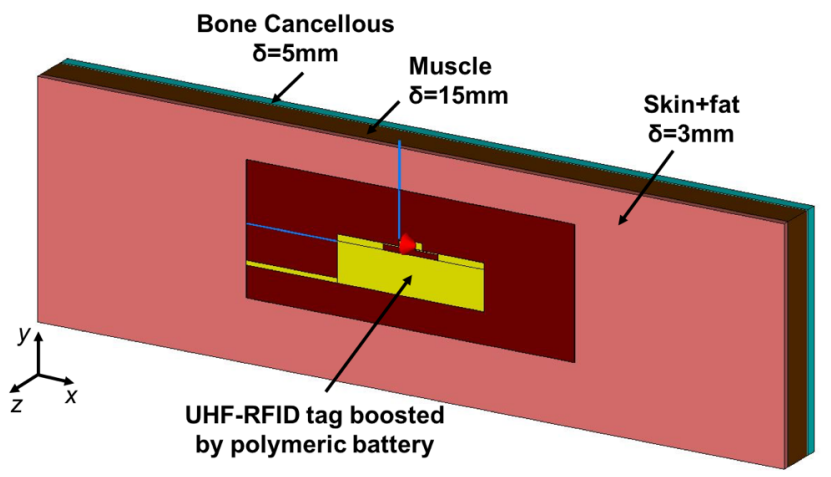

Fig. 2 UHF-RFID antenna boosted by polymer-based battery mounted on multilayer human body model. $\delta$ is the thickness of human tissue.

also designed to connect the $\mathrm{V}_{\text {BATT }}$ pin of the microchip with the battery cathode, while a copper tail was necessary to enable the connections for charging the battery. The external sizes of the tag were fixed to $\mathrm{L}=65 \mathrm{~mm}$ and $\mathrm{W}=20 \mathrm{~mm}$ as a trade-off between a reasonable antenna gain and moderate overall antenna size. The slot shape is instead appropriately sized $(1=24.5 \mathrm{~mm}$, $\mathrm{w}=2.3 \mathrm{~mm})$ in order to match the impedance of the RFID microchip, which is $Z_{\text {chip }}=7.4-\mathrm{j} 122 \Omega$ at $866 \mathrm{MHz}$ in batteryassisted (BAP) mode. The electromagnetic modeling of the radio-sensor also comprised the layered polymeric battery. In particular, the nested slotline antenna was integrated into the battery anode, which was formed of a thin layer of Pedot:PSS. The battery cathode was an aluminum layer. The antennabattery system was simulated on a simplified multilayered human body model (Fig. 2) using the transient solver. The resulting gain was $-16.1 \mathrm{~dB}$ at $870 \mathrm{MHz}$ with the power transfer coefficient $\tau=4 \mathrm{R}_{\mathrm{A}} \mathrm{R}_{\text {chip }} /\left|\mathrm{Z}_{\mathrm{A}}+\mathrm{Z}_{\text {chip }}\right|^{2}=0.65$ at $870 \mathrm{MHz}$. Moreover, it is worth observing that no significant change in electromagnetic parameters vs. frequency of the tag antenna was found when the battery charging process is simulated by adding lithium salt into Pedot:PSS solution. A numerical comparison of the curves of $\tau$ and gain vs. frequency both before and after having simulated the battery cycling process is shown in Fig. 3.
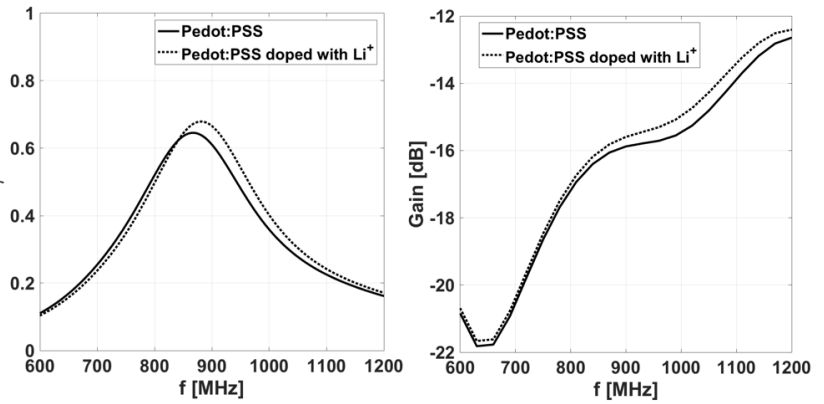

Fig. 3 Simulated power transmission coefficient $\tau$ and gain vs. frequency of the integrated UHF-RFID battery-antenna system on layered human body for Pedot:PSS pure and doped with lithium salt to simulate the battery cycling process. 


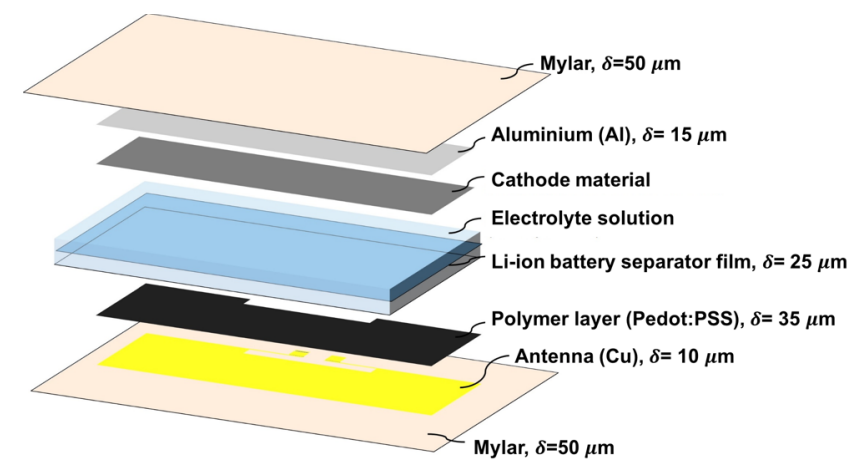

Fig. 4 A sketch of the integrated battery-antenna system.

\section{PRototype AND ON-SKIn PERFoRMANCE}

The multi-layered structure of the integrated battery-antenna system is schematized in Fig. 4. The cathode material for battery was a mixture based on $\mathrm{LiNi}_{0.5} \mathrm{Mn}_{1.5} \mathrm{O}_{4}(80 \% \mathrm{wt})$, Super $\mathrm{P}$ carbon black $(10 \% \mathrm{wt})$ and PVdF $(10 \% \mathrm{wt}$, (Poly (vinylidene fluoride) average $\mathrm{Mw}-534,000$ by $\mathrm{GPC}$, powder, Aldrich) deposited by means of Doctor blades printing on a thin aluminum (Al) layer. A prototype of the radio-sensor was fabricated using conventional wet etching of a copper clad Mylar sheet for the antenna while the different components of the polymeric battery were assembled inside a glovebox filled with Argon gas.

To enable the connection to the battery cathode, a thin wire was soldered to the pad of the chip hosting the battery connection. Capacity, charging voltage and discharge characteristics of the polymeric battery were individually tested and the performance of the radio-sensor integrating battery was investigated on body. The interrogating measurement set-up consisted of the Tagformance UHF RFID reading system by Voyantic connected to $8.15 \mathrm{dBi}$ gain linearly-polarized patch antenna placed at a fixed distance apart from the radio-sensor. The communication performances of the antenna boosted by the polymeric battery were characterized through the measurement of the realized gain $\mathrm{G}_{\tau}$, i.e. the gain of the tag attached directly onto a volunteer's skin scaled by the mismatch to the IC. In particular, by comparison of measurement and numerical simulation (Fig. 5), the maximum measured realized gain is significantly shifted towards lower frequencies with $2 \mathrm{~dB}$ variation at $870 \mathrm{MHz}$ with respect to the simulated profile.

\section{DISCUSSION AND CONCLUSION}

Organic polymer-based conductors are promising for the development of eco-friendly and biocompatible non-metallic thin film batteries that can be integrated within the antenna. The realized integrated polymeric battery-antenna system is flexible and skin-conformable with performance that could be comparable with that of a standard coin cell battery.

Moreover, the microwave characterization of the polymeric films under test enabled the design of a new class of on-skin radio-sensors. However, some critical issues still require investigation and improvement, such as the short battery life (currently approximately 1 hour) and the sealing of the different battery components so as to enable optimal performance.

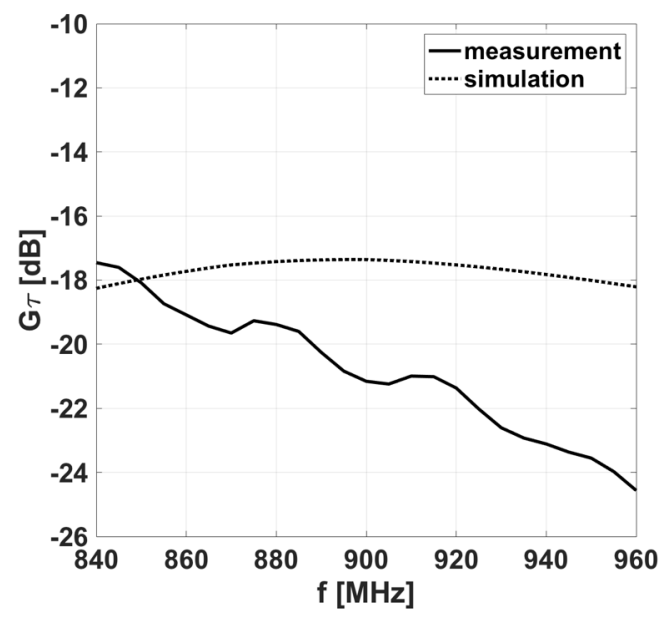

Fig. 5 Measured and simulated realized gain along the antenna axis (broadside observation) vs. frequency.

\section{REFERENCES}

[1] J. Kim, R. Kumar, A. J. Bandodkar, and J. Wang, "Advanced Materials for Printed Wearable Electrochemical Devices: A Review," Advanced Electronic Materials, 2017.

[2] M. Woods and J. Batchelor, "Low-profile Slot Antenna Integrated with a Thin Polymer Non-Metallic Battery," 2013 Loughborough Antennas \& Propagation Conference (LAPC), Loughborough, 2013, pp. 441-445.

[3] S. Muench, A. Wild, C. Friebe, B. Häpler, T. Janoschka, and U. S. Schubert, "Polymer-Based Organic Batteries," Chemical Reviews, 116, 9438-9484, 2016.

[4] S. Amendola, C. Occhiuzzi, A. Ajovalasit, M. A. Sabatino, C. Dispenza and G. Marrocco, "Dielectric characterization of biocompatible hydrogels for application to Epidermal RFID devices," 2015 European Microwave Conference (EuMC), Paris, 2015, pp. 379-382.

[5] M. A. Ziai and J. C. Batchelor, "Temporary On-Skin Passive UHF RFID Transfer Tag," IEEE Transactions on Antennas and Propagation, vol. 59, no. 10 , pp. 3565-3571, Oct. 2011. 